\title{
Click chemistry targets antibody-drug conjugates for the clinic
}

Bioorthogonal chemistry, already a workhorse of drug discovery research, prepares for the leap into human testing.

$\mathrm{R}$ esearchers at the Memorial Sloan Kettering Cancer Center in New York are preparing the first test in humans of a new type of diagnostic that combines a radioisotope and antibody via bioorthogonal 'click' chemistry. The trial is expected to be a proof of principle of the clinical potential of bioorthogonal chemistry-reactions that can be performed in living systems without interfering with the surrounding biology. Over the past two decades, this type of chemistry has been increasingly used by bio-inorganic and medicinal chemists, most notably in the development of targeted medicines, such as antibody-drug conjugates (ADCs). But the Sloan Kettering trial will be first test of such reactions inside human participants.

The researchers will first inject patients with an experimental human IgG1 monoclonal antibody $(\mathrm{mAb}) 5 \mathrm{~B} 1$ that is conjugated to transcyclooctene and targets a carbohydrate antigen (Sialyl-Lewis) on pancreatic ductal adenocarcinoma. Once the conjugated $\mathrm{mAb}$ binds the antigen, a follow-up injection delivers the diagnostic radioisotope copper-64 conjugated to tetrazine, which undergoes a DielsAlder bioorthogonal reaction with the transcyclooctene moiety on the mAb. The resulting bond creates a radio-diagnostic $\mathrm{mAb}$ that marks cancer cells' location for imaging by positron emission tomography (PET). "We're hoping the first patients will be done in the autumn this year," says Jason Lewis, who leads the work.

Meanwhile, several biotech companies are also working on techniques that would shift bioorthogonal reactions from the lab into patients, potentially offering new ways unleash powerful cancer drugs and antibiotics in a controlled way.

"Big pharma is waiting to see the results; it's small biotechs that are moving these new ways of treatment right now," says Asier Unciti-Broceta, who works on bioorthogonal chemistry at the University of Edinburgh, UK.

Bioorthogonal chemistry relies on very rapid, spontaneous reactions between two chemical groups that are not normally found in biology. These two complementary 'handles' can only react with each other, in what is sometimes called a 'click' reaction. "You can reliably run these reactions inside
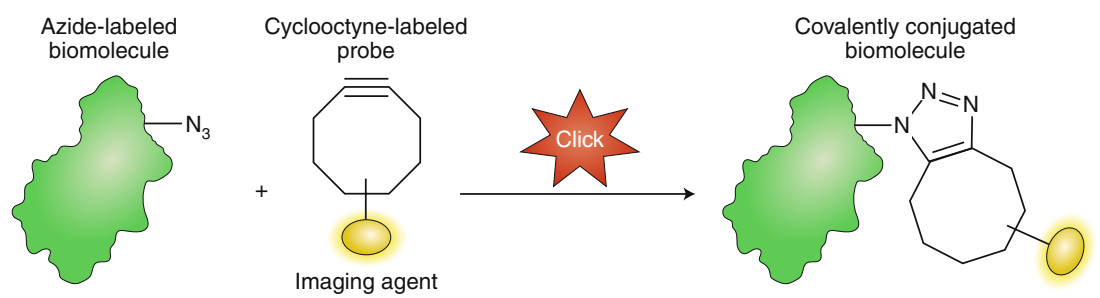

A click chemistry reaction developed by Carolyn Bertozzi takes place between azide and alkyne (here a cyclooctyne) components, and because these groups are not present in biomolecules, it leaves other chemical groups untouched and can be used in living cells.

a cell or an animal," says Neal Devaraj at the University of California San Diego, who has developed bioorthogonal reactions.

"So cells and zebrafish become the new flasks for organic chemistry."

Some of the earliest examples of bioorthogonal reactions emerged from labeling experiments performed in Carolyn Bertozzi's lab at Stanford University. In 2004, Bertozzi optimized an azide and a cyclooctyne reaction to tag cell-surface glycans with fluorescent probes, to map the glycans' distribution and activity. Bertozzi's approach has since become a mainstay of bioorthogonal chemistry, and a fluorescence imaging method widely adopted in drug development labs.

What makes bioorthogonal reactions attractive for in vivo labelling is not only their high speed, even in the complex environment of living cells, but also the ease with which the reagents can penetrate cells. Conventional fluorescence imaging agents are often bulky molecules that may be incompatible with a cell's biochemistry. In contrast, bioorthogonal chemistry enables the imaging agent's two key components-a targeting molecule and a fluorescent molecule-to be introduced sequentially into cell cultures or organisms before being clicked together. The click reaction involves two chemical groups (for example, tetrazine and transcyclooctene) that are not normally found in biology, so they react quickly and exclusively with each other. These bioorthogonal handles also tend to be smaller than either of the molecules they bring together, so they do not prevent the two components from integrating seamlessly into the cells' biochemical reactions. This two-step process ensures that the size of the finished imaging agent does not compromise its ability to be incorporated into cells.

In drug discovery, bioorthogonal chemistry has proven useful in many other ways. For example, once a targeting molecule binds a particular protein, it can be clicked with a partner that carries a biotin molecule. A polymer bead coated with streptavidin-which binds strongly to biotin-can then be used to pull out the entire assembly, including the targeted protein, for further study. "Target discovery using various bioorthogonal methods has really become pretty mainstream in pharma companies," says Bertozzi.

For companies developing ADCs, click chemistry is also providing improvements. To date, the US Food and Drug Administration (FDA) has approved five ADCs, all cancer treatments, including the go-ahead on June 10 for Genentech's Polivy (polatuzumab vedotin-piiq), a humanized anti-CD79b IgG1 mAb conjugated to the antimitotic agent monomethyl auristatin E for the treatment of diffuse large B-cell lymphoma. But according to Bertozzi, Polivy and other first-generation ADCs are actually mixtures of many different biologic conjugates. This is because traditional coupling reactions in $\mathrm{ADC}$ production cannot control how many cytotoxic drug molecules conjugate to amino acid residues in the $\mathrm{mAb}$, and in which precise positions on the $\mathrm{mAb}$. Consequently, the mixture of $\mathrm{ADCs}$ in a manufacturing batch can reduce the efficiency and increase toxicity of the product.

Introducing a bioorthogonal handle on the mAb offers a way to control the number and position of conjugated drug 
Table 1 | Selected biotech companies working with bioorthogonal chemistry for ADC preparation

\begin{tabular}{|c|c|c|c|c|}
\hline Name & ADC components & $\begin{array}{l}\text { Leading candidates } \\
\text { (partner) }\end{array}$ & Target & Progress (date initiated) \\
\hline Ambrx & $\begin{array}{l}\text { HER2 targeting antibody + } \\
\text { Amberstatin269 }\end{array}$ & ARX788 & $\begin{array}{l}\text { Advanced cancers with HER2 } \\
\text { expression }\end{array}$ & Phase 1 (March 2016) \\
\hline $\begin{array}{l}\text { Catalent/ Redwood } \\
\text { Bioscience }\end{array}$ & Anti-CD22 antibody + maytansine & $\begin{array}{l}\text { TRPH-222 (Triphase } \\
\text { Accelerator Corp.) }\end{array}$ & Non-Hodgkin lymphoma & Phase 1 (April 2019) \\
\hline Sutro Biopharma & $\begin{array}{l}\text { Anti-CD74 IgG1 antibody (SP7219) + } \\
\text { maytansine }\end{array}$ & STRO-001 & $\begin{array}{l}\text { Multiple myeloma and non-Hodgkin } \\
\text { lymphoma }\end{array}$ & Phase 1 (April 2018) \\
\hline Sutro Biopharma & $\begin{array}{l}\text { Anti-FolRa human IgG1 antibody } \\
\text { (SP8166) + 3-aminophenyl } \\
\text { hemiasterlin }\end{array}$ & STRO-002 & Ovarian and endometrial cancers & Phase 1 (March 2019) \\
\hline Synaffix & $\begin{array}{l}\text { Anti-AXL IgG1 antibody + } \\
\text { pyrrolobenzodiazepine dimer SG3199 }\end{array}$ & $\begin{array}{l}\text { ADCT-601 (ADC } \\
\text { Therapeutics) }\end{array}$ & Solid tumors with $\mathrm{AXL}$ expression & Phase 1 (January 2019) \\
\hline Syndivia & Undisclosed & SDV1101 & Breast cancer, liposarcoma & $\begin{array}{l}\text { Preclinical: human trials } \\
\text { expected } 2021\end{array}$ \\
\hline Tagworks & $\begin{array}{l}\text { CC49 fragment + monomethyl } \\
\text { auristatin } \mathrm{E}\end{array}$ & TAGW-110 & $\begin{array}{l}\text { Tumor-associated glycoprotein } 72 \\
\text { (TAG72) in various cancers }\end{array}$ & Preclinical \\
\hline
\end{tabular}

molecules, and to identify the most effective ADC formulation. "It allows us to precisely control the drug-antibody ratio," says Sergii Kolodych, chief scientific officer at Syndivia, a biotech company in Illkirch, France. Syndivia is using bioorthogonal chemistry to produce anti-tumor ADCs that have higher efficacy than conventional treatments in mouse models of breast cancer and gastric carcinoma, Kolodych says.

Several other companies are using the approach to create ADCs, some of which are already in phase 1 trials (Table 1). Bertozzi spun out a company called Redwood Bioscience (subsequently acquired by Catalent of Somerset, New Jersey) to commercialize a bioorthogonal coupling technique called SMARTag, and a SMARTag ADC entered clinical trials for the first time in April. The lymphoma treatment, TRPH-222, teams an anti-CD22 antibody with two molecules of maytansine, which inhibits microtubule assembly.

Although click chemistry now has a track record as a research tool, the sheer complexity of living systems means that transferring it into humans poses a formidable challenge. "It is more tricky for those companies hoping to use this chemistry in humans," says Kolodych. Over the past few years, though, radioisotope targeting, either for imaging applications or to kill off tumor cells, has emerged as the leading potential clinical application.

Antibody-radioisotope therapeutics have a long history, with two therapeutic agents approved by the FDA in the early 2000s, but they have been slow to gain wide adoption. One drawback is that the conjugates initially spread throughout the body, seeking out their target, and it can take days before unbound conjugates are completely cleared from the body, which leads to side effects caused by off-target radiation. When such conjugates are used in imaging applications, where many radioisotopes have half-lives of just a few hours, the radiation may have faded substantially before it can clearly reveal the location of target cells.

To avoid this problem, researchers, such as Lewis, have switched to the bioorthogonal strategy. Lewis' tests in mice have shown that this approach is more effective than injecting a pre-prepared $\mathrm{mAb}$-radioisotope conjugate. He has used the method with various radioisotopes, including actinium-225, which delivers a tumor-killing blast of alpha particles, and fluorine-18, which releases positrons for PET imaging. "I'm surprised it's worked so well, it's been incredibly efficient," he says. His group has settled on another positron emitter, copper-64, which has a 13-hour half-life, for their human trial in pancreatic ductal adenocarcinoma. The team has completed its preclinical work and plans to submit its Investigational New Drug (IND) application to the FDA this September. The NIH-funded phase 1 trial will study the safety and biodistribution of the agent in ten patients. If successful, the team hopes to move quickly to similar trials using actinium-225 and fluorine-18. "The possibilities are enormous," says Lewis. The technique should reduce the radiation dose that patients receive from the imaging isotope, he says, and splitting the process into two stages will also make it easier to schedule the time-sensitive injection of the freshly prepared radioisotope. "There is enormous interest in this from pharma now."

In Nijmegen, the Dutch company Tagworks Pharmaceuticals has also pioneered the pre-targeting approach for in vivo imaging and radiotherapy with lutetium-177, and hopes to translate its mouse studies into humans soon, says Marc Robillard, CEO of Tagworks. The company is also part of a European Union consortium, Click-It, which is developing a wider range of bioorthogonal handles for radioimaging applications that use shortlived isotopes such as fluorine-18.

Aside from radioisotopes, Tagworks is developing a bioorthogonal strategy to overcome some of ADC's shortcomings in targeting solid tumors. Conventional ADCs must be internalized by the cells, and once inside, they are broken down by enzymes to release their cytotoxic agent. But Robillard says that there are relatively few tumorspecific receptors that can efficiently bring ADCs into the cells.

Tagworks is instead using a 'clickto-release' approach to avoid that internalization step. The company's ADCs target non-internalizing receptors, and contain a linker that can only be broken by a bioorthogonal reaction. Once the conjugate has bound to its target on the outside of a cell, an activating reagent is administered to release the drug, which is then taken up by tumor cells. Last year Tagworks unveiled the first in vivo example of a therapy that used bioorthogonally triggered drug release from a targeting agent. The treatment shrank tumors and extended survival in mice with colorectal or ovarian tumor grafts. The company is now selecting a lead compound to take forward into human trials.

Shasqi of San Francisco, California, is also aiming to run bioorthogonal reactions inside patients, but is using a very different 
targeting mechanism. Researchers inject an alginate hydrogel modified with bioorthogonal handles (tetrazines) at the site where a drug is needed, and then add a pro-drug that carries complementary handles (for example, trans-cyclooctene modified doxorubicin). The pro-drug is stable and almost inactive in the body, until it comes into contact with the hydrogel, at which point the bioorthogonal reaction frees the drug. This reduces off-target exposure and enables very high local dosing of the drug.

The company has used the method to deliver doxorubicin in mice with soft tissue sarcoma grafts and found that it causes substantially lower side effects and greater efficacy than treatment with doxorubicin alone. Similar experiments in mice using an antibiotic pro-drug showed that the hydrogel contained enough bioorthogonal handles to activate the antibiotic even after repeated doses. "That's because the amount of binding agent you have on the material is so much larger than the dose you're providing," explains Jose M. Mejia Oneto, President and CEO of Shasqi.

Unciti-Broceta hopes that the launch of first clinical trials of in vivo bioorthogonal chemistry will generate more interest in this multidisciplinary field. Meanwhile, specialist companies are now providing the starting reagents needed for bioorthogonal chemistry, making it more accessible for those unfamiliar with organic synthesis. "The feeling is that the community is really growing," Unciti-Broceta says, "and it's helping to create a lot of new collaborations."

\section{Mark Peplow}

Cambridge, UK

Published online: 5 July 2019

https://doi.org/10.1038/d41587-019-00017-4

\section{PODCAST}

\section{First Rounders: Cigall Kadoch}

Cigall Kadoch is a co-founder of Foghorn Therapeutics and an assistant professor at the Dana-Farber Cancer Institute, where she runs the Kadoch lab. In her talk with Nature Biotechnology, she discusses launching Foghorn, how geography affects biotech success, and the link between interior design and scientific rigor. https://www.nature.com/nbt/podcast

Published online: 2 August 2019

https://doi.org/10.1038/s41587-019-195-y 